\title{
Deep Vein Thrombosis is Modulated by Inflammation Regulated via Sirtuin 1/NF-KB Signalling Pathway in a Rat Model
}

\author{
Xiaolan Yao ${ }^{1}$ Wenpei Chen ${ }^{1}$ Jin Liu ${ }^{1}$ Han Liu ${ }^{1}$ Janis Y. Zhan ${ }^{1}$ Shixia Guan ${ }^{1}$ Ziqi Lu ${ }^{1}$ Ping Tang ${ }^{1}$ \\ Peng $\mathrm{Li}^{1}$ Baoqin $\mathrm{Lin}^{1}$ \\ ${ }^{1}$ School of Pharmaceutical Sciences, Guangzhou University of \\ Chinese Medicine, Guangzhou, China \\ Thromb Haemost 2019;119:421-430.

\begin{abstract}
Address for correspondence Baoqin Lin, PhD, School of Pharmaceutical Sciences, Guangzhou University of Chinese Medicine, 232 Wai Huan East Road, Guangzhou University Town, Panyu District, Guangzhou 510006, China (e-mail: linbaoqin@gzucm.edu.cn).
\end{abstract}

Abstract




Keywords
- deep vein thrombosis
- SIRT1
- inflammation
- resveratrol
- EX527

Background Inflammation plays an important role in thrombus formation, and Sirtuin 1 (SIRT1) negatively regulates inflammation via deacetylating nuclear factorkappa B. However, the relationship between SIRT1-regulated inflammation and deep vein thrombosis (DVT) is still unknown.

Objective The aim of this study was to investigate whether SIRT1 plays a critical role in inferior vena cava (IVC) stenosis-induced DVT.

Materials and Methods Thrombus weight and histopathologic analysis of IVC were evaluated at different time points after IVC stenosis in rats. Serum levels of inflammatory cytokines and protein expressions of SIRT1, acetylated p65 (Ace-p65), phosphorylated p65 (p-p65) and tissue factor (TF) in thrombosed IVC were assessed. Besides, the effects of resveratrol (RES, a SIRT1 agonist) and EX527 (a selective SIRT1 inhibitor) on DVT were evaluated.

Results Thrombus weight was increased from 1 to 3 days after IVC stenosis, and then was decreased afterwards. Leukocytes infiltration appeared and serum levels of cytokines were significantly increased in rats of IVC stenosis. SIRT1 protein expression was significantly down-regulated at 1 hour and 1 day after stenosis, while p-p65, Acep65 and TF protein expressions appeared a contrary trend. RES reduced thrombus weight, leukocytes infiltration, levels of tumour necrosis factor- $\alpha$ and interleukin- $1 \beta$ and protein expressions of Ace-p65 and TF as well. Moreover, RES significantly increased the protein and messenger ribonucleic acid expressions of SIRT1, while EX527 abolished the protective effects of RES.

Conclusion SIRT1 activation attenuated IVC stenosis-induced DVT via anti-inflammation in rats. Therefore, SIRT1 may be a potential therapeutic target that could ameliorate DVT.

\section{Introduction}

Deep vein thrombosis (DVT) is one of the leading causes of morbidity and mortality worldwide owing to the development of pulmonary embolism, a fatal sequela that occurs when the thrombus breaks apart and moves to the lung. ${ }^{1}$ DVT is common after surgery, especially after hip or knee arthroplasty. Morbidity of DVT after hip or knee arthroplasty reaches to 35\% without a post-operative prescription anticoagulation claim. ${ }^{2}$ Inflammation plays a pivotal role in thrombosis. ${ }^{3-7}$ Our previous study also showed a close relationship between DVT received

September 7, 2018

accepted after revision

November 18, 2018
DOI https://doi.org/

10.1055/s-0038-1676987. ISSN 0340-6245. (c) 2019 Georg Thieme Verlag KG Stuttgart · New York
License terms

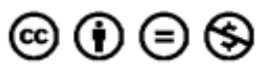


and inflammation. ${ }^{8}$ Neutrophils, monocytes and lymphocytes exert critical roles in thrombus formation. Adhesion of neutrophils has been identified as the initial event during the process of thrombosis. ${ }^{9}$ Serine protease and elastase, released by neutrophils, cleave and oxidize anticoagulants including tissue factor pathway inhibitor and thrombomodulin (TM). Moreover, neutrophil extracellular traps (NETs) bind to von Willebrand factor and then activate platelets. Monocytes participate in the processes of thrombosis and inflammation by expressing tissue factor (TF) and P-selectin, respectively. ${ }^{10}$ Leukocyte-released pro-inflammatory cytokines such as interleukin-1 $\beta$ (IL-1 $\beta$ ) and tumour necrosis factor- $\alpha$ (TNF- $\alpha$ ) promote thrombosis via down-regulating endothelial protein $C$ receptor and $\mathrm{TM}^{9}{ }^{9}$ Intriguingly, all of these molecules are regulated by nuclear factor-kappa $\mathrm{B}(\mathrm{NF}-\mathrm{kB}) .^{11,12}$

Yeast silent information regulator 2 (Sir2) is a nicotinamide adenine dinucleotide-dependent protein with deacetylase and adenosine diphosphate-ribosyltransferase activities. Mammalian homologues of Sir2 are made up of seven members, Sirtuin (SIRT) 1-7. SIRT1-7 are implicated in a variety of cellular functions such as gene silencing, cell apoptosis and energy homeostasis in the nucleus, cytosol and mitochondria. ${ }^{13}$

SIRT1 is the closest homologue to Sir2 and it is essential for cell survival, differentiation, senescence and metabolism via anti-inflammation or antioxidation. ${ }^{14}$ SIRT1 deacetylates p65 at Lys310, which results in transcriptional silencing at facultative heterochromatin of pro-inflammatory genes such as TNF- $\alpha$ and IL-1 $\beta .^{15,16}$ It has been reported that SIRT1 inhibition increases TF activity and promotes arterial thrombosis in vivo. ${ }^{17}$ SIRT3 deficiency does not affect venous thrombosis or NETosis despite mild elevation of intracellular reactive oxygen species in platelets and neutrophils in mice. ${ }^{18}$ However, no evidences have shown whether regulation of inflammation by SIRT1 is involved in DVT.

To assess the relationship among SIRT1/NF-KB signalling pathway, inflammation and DVT, serum levels of inflammatory cytokines, and morphological changes, leukocytes infiltration, protein expressions of SIRT1, phosphorylated p65 (p-p65) and acetylated p65 (Ace-p65) of thrombosed inferior vena cava (IVC) were evaluated in rats of stenosis-induced DVT. Resveratrol (RES) and EX527, the agonist and antagonist of SIRT1, were applied to further determine the role of SIRT1 in DVT.

\section{Materials and Methods}

\section{Animals}

Male and female Sprague-Dawley (SD) rats (250-300 g, approved NO. SCXK 2013-0034) were provided by the Experimental Animal Center, Guangzhou University of Chinese Medicine. All rats had free access to a standard diet and drinking water, and were housed in a room at $24.0 \pm 0.5^{\circ} \mathrm{C}$ and with a 12-hour/12-hour cyclic lighting schedule. The experiments were performed in compliance with the Animal Ethics Committee of Guangzhou University of Chinese Medicine.

\section{IVC Stenosis-Induced DVT Model in Rats}

IVC thrombus was induced as described previously with slight modification. ${ }^{8}$ In brief, rats were anaesthetized with intraper- itoneal injection of pentobarbital sodium (36 mg/kg, Merck, Darmstadt, Germany) and then a 2-cm incision was performed along the abdominal midline. The IVC just caudal to the left renal vein was partially ligated by tying a $5-0$ silk suture tightly around the vein together with an acupuncture pin $(0.35 \mathrm{~mm}$ $\times 50 \mathrm{~mm}$ ) and then removing the pin. The abdominal muscle and skin were sutured. After closing each layer, ceftriaxone sodium (Lijian, Shenzhen, China) was sprinkled evenly along the incision line to prevent bacterial infection. The rats in the sham group received the same surgical procedure without IVC stenosis.

\section{Study Design}

This study was composed of three parts. In the first part, the blood and thrombosed IVC were harvested at 0.5 hour, 1 hour, 1 day, 3 days, 5 days, 7 days or 14 days after stenosis. In the second part, RES was applied to investigate the effect of SIRT1 on thrombus formation. Rats were randomly divided into five groups: sham group, model group, low, medium and high doses of RES (Sigma-Aldrich, Missouri, United States) groups. RES was dissolved in $0.5 \%$ sodium carboxymethyl cellulose, and orally administered at 25,50 and $75 \mathrm{mg} / \mathrm{kg}$ once daily for 2 days. The first dose was administered at 1 hour before thrombus induction, and the second one was at 24 hours after surgery. The rats in the sham and model groups were orally administered with vehicle.

In the third part, rats were randomly divided into eight groups: (1) normal group; (2) sham group; (3) sham + RES group; (4) sham + EX527 group; (5) model group; (6) model + RES group; (7) model + RES + EX527 group; and (8) model + EX527 group. RES was orally administered at $50 \mathrm{mg} / \mathrm{kg}$ once daily for 2 days. The first dose was administered at 1 hour before thrombus induction, and the second one was at 24 hours after surgery. EX527 (10 mg/kg, Selleck Chemicals, Texas, United States) was dissolved in a solution of $1 \%$ dimethyl sulfoxide, $30 \%$ polyethylene glycol 400 and $0.9 \%$ sodium chloride, and intraperitoneally injected in a volume of $2 \mathrm{~mL} / \mathrm{kg}$ body weight 20 minutes before thrombus induction. ${ }^{19,20}$

\section{Thrombus Weight}

At the indicated time points or 1 hour after the final administration of RES, rats were anaesthetized with pentobarbital sodium. Blood samples from the carotid artery were collected into ordinary vacuum blood collection tubes without any anticoagulants or coagulants. IVCs were then dissected, weighed and split into two parts. One part was fixed in $4 \%$ paraformaldehyde dissolved in phosphate-buffered saline (PBS) ( $\mathrm{pH} \mathrm{7.4)} \mathrm{for} 48$ hours at $4^{\circ} \mathrm{C}$ for histopathologic and immunohistochemical analysis. And the other part was stored at $-80^{\circ} \mathrm{C}$ for Western blot, quantitative real-time polymerase chain reaction (qRT-PCR) and immunofluorescence analysis.

\section{Measurement of Inflammatory Cytokines}

Sixty minutes after blood collection, the serum was obtained by centrifugation at $1,600 \times \mathrm{g}$ for 10 minutes at $4^{\circ} \mathrm{C}$, stored at $-80^{\circ} \mathrm{C}$ and detected within 2 weeks. Serum levels of IL-1 $\beta$ (Invitrogen, California, United States) and TNF- $\alpha$ (Lianke 
Biotech, Hangzhou, China) were assayed using commercially available enzyme-linked immunosorbent assay kits.

\section{Histopathology and Immunohistochemistry}

IVCs fixed in 4\% paraformaldehyde were dehydrated through graded alcohol, mounted in paraffin wax and sectioned into slices of $4 \mu \mathrm{m}$ thickness. Specimens were stained with haematoxylin and eosin. The numbers of neutrophils, monocytes and lymphocytes within the vein wall and thrombus of each sample were counted and summed from five high power fields $(\times 400) .{ }^{21}$ For immunohistochemical staining, specimens were immersed in $3 \% \mathrm{H}_{2} \mathrm{O}_{2}$ for 10 minutes, blocked with $10 \%$ goat serum and then incubated with antibody to TF (1:50; Abcam, Cambridge, United Kingdom) at $4^{\circ} \mathrm{C}$ overnight. Sections were then processed using a rabbit and mouse horseradish peroxidase (HRP) kit (CWBIO, Beijing, China). Pathological changes in thrombosed IVCs were observed under an optical microscope (BX53; Olympus, Tokyo, Japan).

\section{Western Blot Analysis}

IVCs taken from $-80^{\circ} \mathrm{C}$ fridge were lysed with solution containing $98 \%$ radioimmunoprecipitation assay buffer, $1 \%$ phenylmethylsulfonyl fluoride and $1 \%$ phosphatase inhibitor (CWBIO). After homogenization on ice, tissue lysates were centrifuged at $14,000 \times \mathrm{g}$ for 10 minutes at $4^{\circ} \mathrm{C}$ and the supernatants were then obtained. Protein concentrations were measured with a bicinchoninic acid assay protein assay kit (CWBIO). Denatured proteins were separated on $8 \%$ polyacrylamide gels and then transferred onto polyvinylidene difluoride membranes (Millipore, California, United States). After blocking with 5\% bovine serum albumin for 1 hour, the membranes were probed with primary antibodies against rat SIRT1 and Ace-p65 (Lys310) (1:8,000 and 1:250, respectively; Abcam), p65, p-p65 and glyceraldehyde 3-phosphate dehydrogenase (GAPDH) $(1: 1,000$; Cell Signaling Technology, Massachusetts, United States) overnight at $4^{\circ} \mathrm{C}$. Afterwards, HRP-conjugated secondary antibody (Cell Signaling Technology, 1:2,000) was applied. Immunoreactive bands were visualized using a chemiluminescence apparatus (5200CE; Tanon, Shanghai, China), and analysed using the Labwork image analysis software (National Institutes of Health, Maryland, United States).

\section{qRT-PCR Assay}

Total ribonucleic acids (RNAs) were extracted from frozen IVCs using TRIzol (CWBIO). The purity of RNA samples was checked by NanoDrop 2000 spectrophotometer (Thermo Fisher Scientific, Massachusetts, United States). Subsequently, RNA was reversely transcribed into complementary deoxyribonucleic acid (cDNA) using the RevertAid first strand cDNA synthesis kit (Thermo Fisher Scientific). The qRT-PCR was performed with PowerUp SYBR Green Master Mix (Thermo Fisher Scientific). The PCR was performed using ABI 7500 Fast Real-Time PCR system (Thermo Fisher Scientific) as the following: 2 minutes at $50^{\circ} \mathrm{C}$ for uracil- $\mathrm{N}$ glycosylase incubation, 2 minutes at $95^{\circ} \mathrm{C}$ for activation of Dual-Lock DNA polymerase and then 15 seconds at $95^{\circ} \mathrm{C}$,
15 seconds at $60^{\circ} \mathrm{C}$ and 1 minute at $72^{\circ} \mathrm{C}$ for 40 cycles of denature. Gene expression values were calculated by $2^{-\Delta \Delta C T}$ relative expression method. The following primers were used:

(1) SIRT1

Forward: 5-TTGACCTCCTCATTGTTATTGGG-3. Reverse: 5-GGCATACTCGCCACCTAACC-3.

(2) GAPDH

Forward: 5-GGCACAGTCAAGGCTGAG AATG-3. Reverse: 5-ATGGTGGTGAAGACGCCAGTA-3.

\section{Immunofluorescence Analysis}

The frozen IVCs were sectioned into slices of $6 \mu \mathrm{m}$ thickness. Specimens were fixed with cold acetone for 15 minutes at $20^{\circ} \mathrm{C}$ and rinsed three times with PBS containing 0.05\% Tween 20. Sections were permeabilized with $1 \%$ Triton X100 (Ameresco, Massachusetts, United States) for 20 minutes and blocked with $10 \%$ goat serum for 60 minutes. Thereafter, sections were incubated with anti-SIRT1 antibody (1:2,000; Abcam) overnight at $4^{\circ} \mathrm{C}$. After washing with PBS, sections were incubated with a goat anti-mouse secondary antibody conjugated to Alexa Fluor 555 (Cell Signaling Technology) for 60 minutes. Sections were then rinsed with PBS containing $0.05 \%$ Tween 20 and counterstained with 4',6 diamidino-2phenylindole dihydrochloride (Sigma-Aldrich) for 5 minutes. Representative fluorescence images were obtained using a laser scanning confocal microscope (LSM800; Carl Zeiss, Jena, Germany).

\section{Statistical Analysis}

Data were expressed as mean \pm standard error of the mean. The groups were compared using a one-way analysis of variance followed by Tukey's post hoc test with the GraphPad Prism version 5.0 (GraphPad Software Inc., California, United States). $p$-Value of $<0.05$ was considered statistically significant.

\section{Results}

\section{Time Course of IVC Thrombosis}

A red blood filament was observed at 1 hour after stenosis, and a fragile thrombus was formed at 1 day. The thrombus grew into a hard one with time. As shown in - Fig. 1, IVC stenosis induced DVT in a time-dependent manner. Male and female rats exhibited similar thrombi after stenosis (- Supplementary Fig. S1, available in the online version). In terms of thrombus weight and morphological changes, there were no differences among normal and various sham groups (- Supplementary Fig. S2, available in the online version).

\section{Leukocytes Infiltration and TF Expression}

The number of neutrophils reached a maximum at 1 hour and 1 day in the vein wall, while at 1 day in the thrombus. Monocytes and lymphocytes were significantly increased during 1 to 14 days ( $p<0.05$ or $p<0.01$ vs. normal control, Fig. 2A-C). As shown in Fig. 2D, TF was little expressed in normal and 0.5 hour groups, while it was highly expressed in active monocytes, neutrophils and endothelium 1 hour to 14 days after stenosis. 

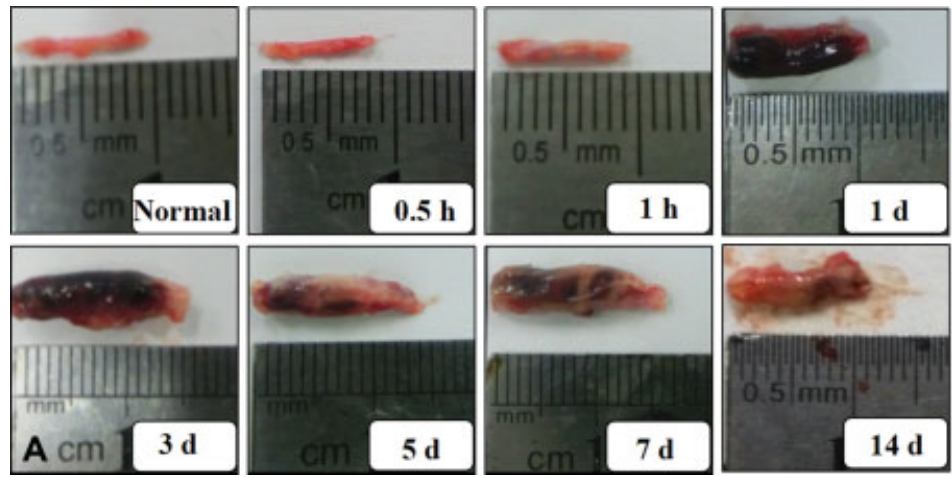

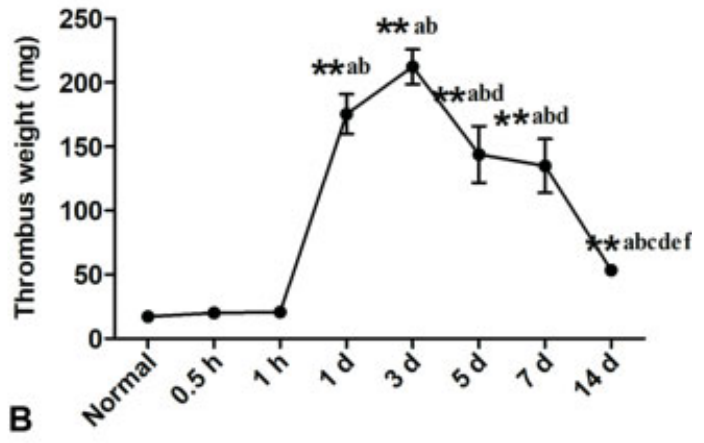

Fig. 1 Deep vein thrombosis (DVT) was induced by inferior vena cava (IVC) stenosis in Sprague-Dawley (SD) rats. At different time points after surgical operation, IVCs were harvested and weighed. One representative IVC of each group (A) and thrombus weight (B) are shown. Data were expressed as mean \pm standard error of the mean (SEM), $n=8 .{ }^{* *} p<0.01$ versus normal group; ${ }^{\mathrm{a}} p<0.05$ versus 0.5 hour group; ${ }^{\mathrm{b}} p<0.05$ versus 1 hour group; ${ }^{c} p<0.05$ versus 1 day group; ${ }^{d} p<0.05$ versus 3 days group; ${ }^{\mathrm{e}} p<0.05$ versus 5 days group; ${ }^{f} p<0.05$ versus 7 days group.

\section{Inflammatory Cytokines}

As illustrated in - Fig. 3, serum levels of IL-1 $\beta$ and TNF- $\alpha$ were elevated by 73.0 and $41.5 \%$ at 1 day after stenosis, respectively ( $p<0.01$ vs. normal group).

\section{Protein Expressions of SIRT1 and NF-kB in IVC}

SIRT1 protein expression was down-regulated by $75.4 \%$ at 1 day after stenosis ( $p<0.05$ vs. normal group, - Fig. 4A). In contrast, p-p65 and Ace-p65 protein expressions were upregulated by 185.4 and $92.4 \%$ at 1 day after surgery, respectively ( $p<0.05$ vs. normal group, - Fig. 4B and C). The protein expressions of SIRT1 (-Fig. 4A) and Ace-p65 (-Fig. 4C) restored towards normal level roughly 3 to 14 days after stenosis, while p-p65 protein expression (-Fig. 4B) recovered towards normal level 14 days after stenosis.

\section{RES Inhibited Thrombosis and Inflammation}

RES inhibited thrombus formation (-Fig. 5) and the infiltration of neutrophils and monocytes in the vein wall and thrombus ( $\mathbf{- F i g . ~ 6 A}$ and $\mathbf{B}$ ). Moreover, RES restored serum IL-1 $\beta(p<0.05$ vs. model group, -Fig. 6C) and TNF- $\alpha$ ( $p<0.05$ vs. model group, - Fig. 6D) towards normal level.

\section{Effects of RES on SIRT1/NF-kB Signalling Pathway}

As shown in - Fig. 7A, RES enhanced messenger RNA (mRNA) and protein expressions of SIRT1. RES at $50 \mathrm{mg} / \mathrm{kg}$ increased SIRT1 protein and mRNA expressions by 420.0 and $45.8 \%$, respectively ( $p<0.05$ vs. model group, - Fig. 7B and C). In contrast, RES at $50 \mathrm{mg} / \mathrm{kg}$ restored Ace-p65 protein expression towards normal level ( - Fig. 7D) and down-regulated TF protein expression by $28.1 \% \quad(p<0.05$ vs. model group, -Fig. 7E).

\section{EX527 Abolished the Protective Effect of RES on Thrombosis}

As shown in - Fig. 8, there were no differences in thrombus weight and protein expressions of SIRT1, Ace-p65 and p-p65 between the normal and sham groups. RES and EX527 had no effect on sham-operated rats. Moreover, EX527 alone had no influence on the above parameters in stenosis-treated rats.
However, EX527 significantly reversed the effect of RES in stenosis-operated rats.

\section{Discussion}

Recently, it has been strongly suggested that inflammation contributes to venous thrombosis. ${ }^{22}$ During the occurrence of DVT, endothelium is active and expresses adhesion molecules that bind to P-selectin glycoprotein ligand-1 or integrin receptor of leukocytes. Active leukocytes and endothelium secret pro-inflammatory cytokines such as IL- $1 \beta$ and TNF- $\alpha$ that inhibit the anticoagulant system. ${ }^{23-25}$ In turn, TNF- $\alpha$ promotes the infiltration of leukocytes. ${ }^{26}$ Cytokines induce leukocytes and endothelial cells to express TF and vice versa. $^{27}$ IVC stenosis induced thrombosis (-Fig. 1), and increased infiltration of leukocytes, TF expression (-Fig. 2) and serum levels of cytokines (-Fig. 3), which may suggest that DVT is associated with inflammation.

It has been reported that SIRT1 knockdown promotes adipose tissue inflammation by controlling the gain of proinflammatory transcription in SD rats, ${ }^{28}$ while SRT1720 (SIRT1 agonist) inhibits amyloid- $\beta$-induced inflammation and retinal pigment epithelial (RPE) barrier disruption via NF-KB pathway in RPE cells. ${ }^{29}$ Our results showed that SIRT1 protein expression within IVC was significantly decreased at 1 hour and 1 day after stenosis, while proteins of Ace-p65 and p-p65 and serum levels of inflammatory cytokines appeared a contrary trend. These results suggest that inhibition of SIRT1 results in inflammation and leads to DVT at the early period of thrombosis.

At 1 and 3 days after stenosis, the thrombus weight was increased to a maximum. However, inflammatory parameters such as neutrophils influx, TNF- $\alpha$ serum level and protein expressions of Ace-p65 and p-p65 at 1 day were much higher than those at 3 days. Moreover, SIRT1 protein expression was decreased to a minimum at 1 day. Therefore, 1 day was chosen as the suitable time point for further research.

RES is an agonist of SIRT1, and exerts various activities such as antioxidation, ${ }^{30}$ anti-inflammation ${ }^{31}$ and anti-apoptosis. ${ }^{32}$ RES at the dose of $5 \mathrm{~g} /$ day has been shown to be safe in 

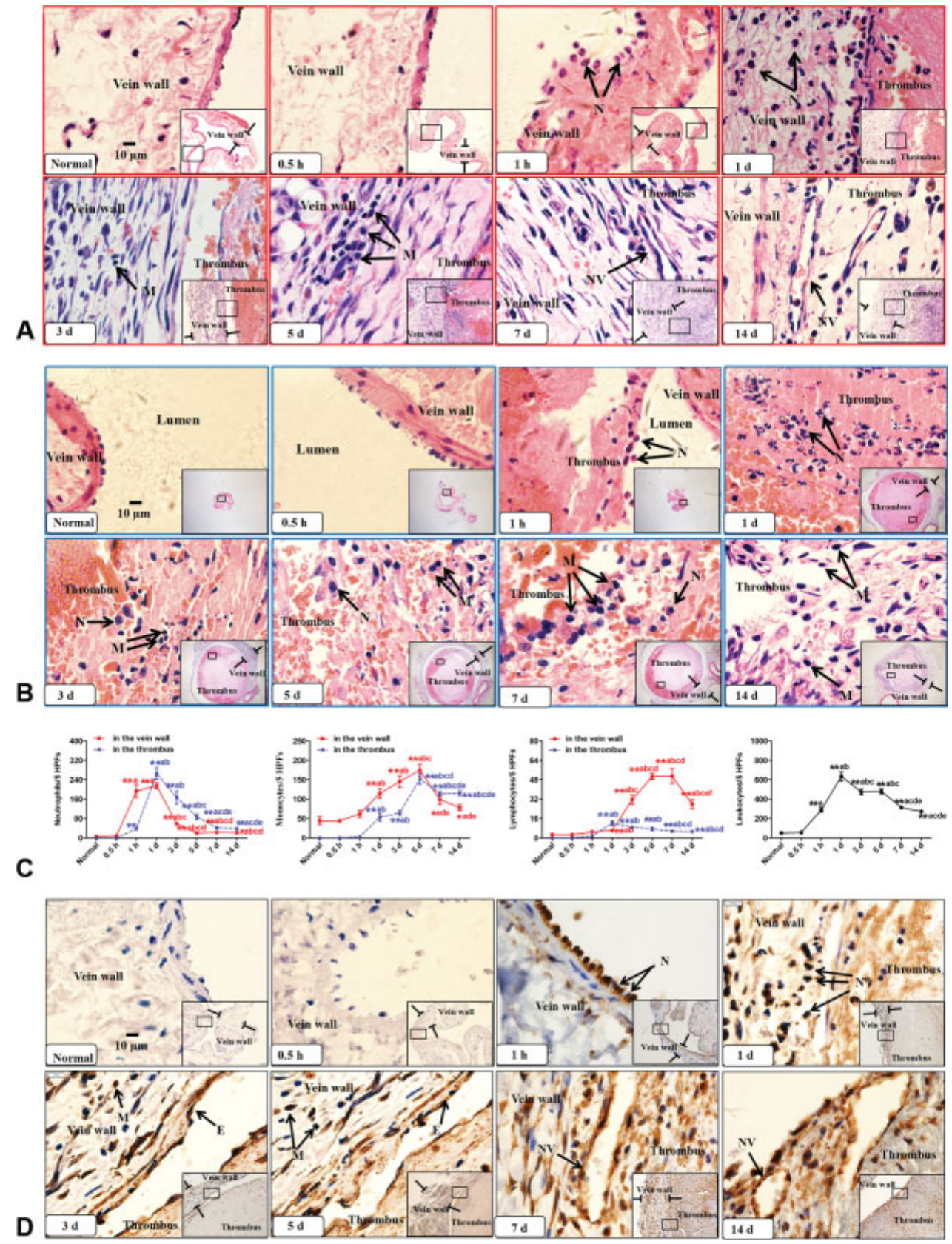

Fig. 2 Inferior vena cava (IVC) stenosis induced leukocytes infiltration and tissue factor (TF) expression in rats. Deep vein thrombosis (DVT) was induced by IVC stenosis in Sprague-Dawley (SD) rats. At different time points after surgical operation, IVCs were collected for histopathological analysis. Boxed region showed representative low magnification and inset in boxed region illustrated higher magnification. (A) Representative haematoxylin and eosin (H\&E) staining of the thrombosed IVC and leukocytes infiltration in the vein wall are shown. (B) Representative H\&E staining of the thrombosed IVC and leukocytes infiltration in the thrombus are shown. (C) Numbers of neutrophils, monocytes and lymphocytes in the vein wall and thrombus, and total numbers of leukocytes in both the vein wall and thrombus are shown. (D) Representative immunohistochemical staining of tissue factor (TF) in the thrombosed IVC. Abbreviations: E, endothelium; L, lymphocyte; M, monocyte; N, neutrophil; NV, new vessel. Data were expressed as mean \pm standard error of the mean (SEM), $n=8$ for A-C, $n=4$ for D. ${ }^{*} p<0.05$ and ${ }^{* *} p<0.01$ versus normal group; a $p<0.05$ versus 0.5 hour group; ${ }^{b} p<0.05$ versus 1 hour group; ${ }^{c} p<0.05$ versus 1 day group; ${ }^{d} p<0.05$ versus 3 days group; ${ }^{\mathrm{e}} p<0.05$ versus 5 days group; ${ }^{\mathrm{f}} p<0.05$ versus 7 days group. Scale bar $=10 \mu \mathrm{m}$.

a clinical study in human. ${ }^{33}$ Therefore, $75 \mathrm{mg} / \mathrm{kg}$ RES in our study may be safe for rats. RES at $50 \mathrm{mg} / \mathrm{kg}$ reduced thrombus weight, leukocytes infiltration and serum levels of cytokines, and increased the expressions of SIRT1 and Ace-p65. These results indicate that up-regulation of SIRT1 inhibits both inflammatory response and DVT. Our results showed that RES at $25 \mathrm{mg} / \mathrm{kg}$ had no effect on inhibiting thrombosis, while another research reported that $25 \mathrm{mg} / \mathrm{kg}$ RES augmented the expression of SIRT1. ${ }^{34}$ The reason may be that RES was intra-gastrically administered in our experiment, whereas it was intraperitoneally injected in their study.

Surgical operation had no effect on protein expression of p-p65 (-Fig. 8), which indicates that surgical trauma has little effect on inflammation. Therefore, RES administration 


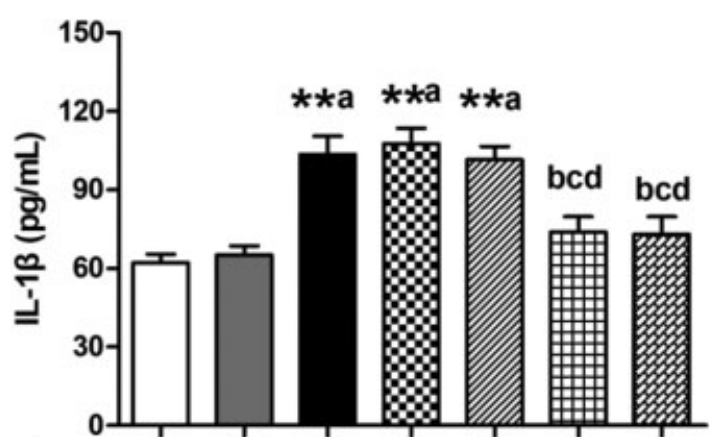

A Normal $0.5 \mathrm{~h} \quad 1 \mathrm{~h} \quad 1 \mathrm{~d} \quad 3 \mathrm{~d} \quad 7 \mathrm{~d} \quad 14 \mathrm{~d}$

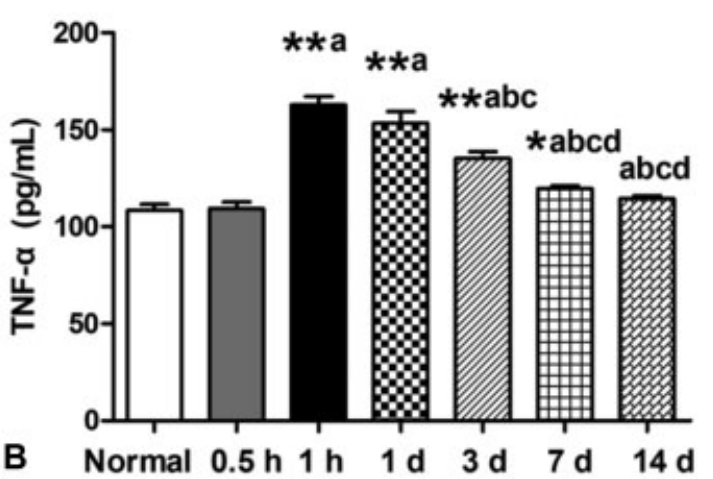

Fig. 3 Serum levels of cytokines in rats of inferior vena cava (IVC) stenosis-induced deep vein thrombosis (DVT). At the indicated time points, whole blood samples were collected and centrifuged to obtain serum for determining levels of (A) interleukin-1 $\beta$ (IL-1 $\beta$ ) and (B) tumour necrosis factor- $\alpha$ (TNF- $\alpha$ ). Data were expressed as mean \pm standard error of the mean (SEM), $n=8 .{ }^{*} p<0.05$ and ${ }^{* *} p<0.01$ versus normal group; ${ }^{\mathrm{a}} p<0.05$ versus 0.5 hour group; ${ }^{\mathrm{b}} p<0.05$ versus 1 hour group; ${ }^{\mathrm{c}} p<0.05$ versus 1 day group; ${ }^{\mathrm{d}} p<0.05$ versus 3 days group.
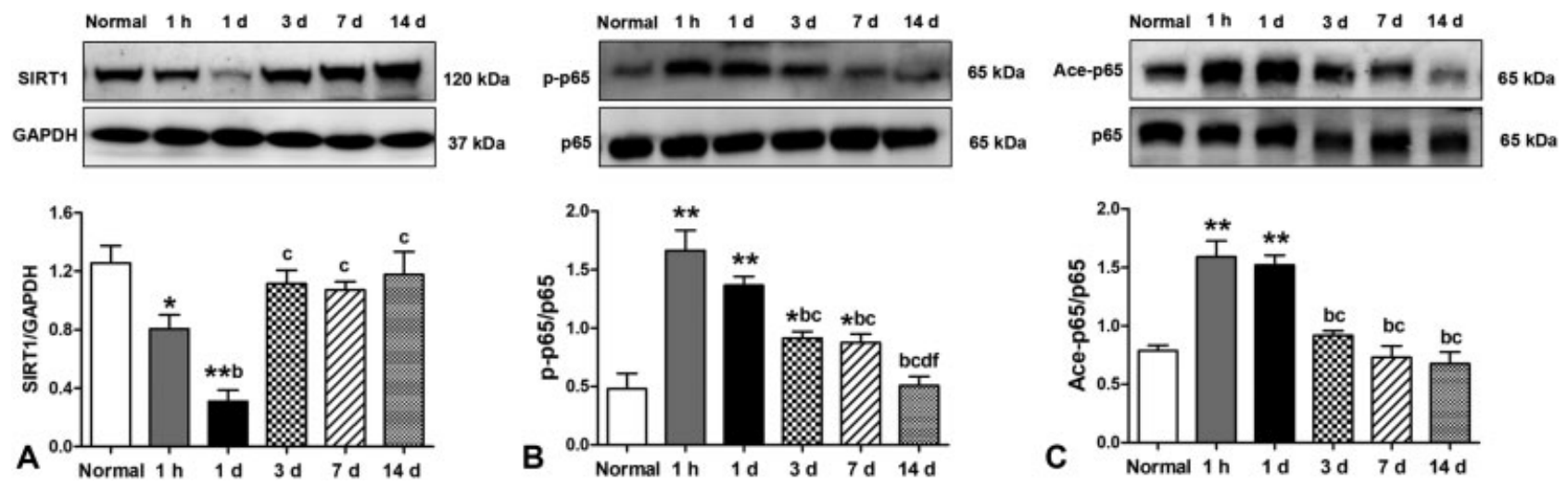

Fig. 4 Protein expressions of Sirtuin 1 (SIRT1), phosphorylated p65 (p-p65) and acetylated p65 (Ace-p65) in rats of inferior vena cava (IVC) stenosis-induced deep vein thrombosis (DVT). At different time points after surgical operation, IVCs were collected for analyses of (A) SIRT1, (B) p-p65 and (C) Ace-p65 protein expressions by Western blot. Data were expressed as mean \pm standard error of the mean (SEM), $n=4 .{ }^{*} p<0.05$ and ${ }^{* *} p<0.01$ versus normal group; ${ }^{b} p<0.05$ versus 1 hour group; ${ }^{c} p<0.05$ versus 1 day group; ${ }^{d} p<0.05$ versus 3 days group; ${ }^{\text {f }} p<0.05$ versus 7 days group.

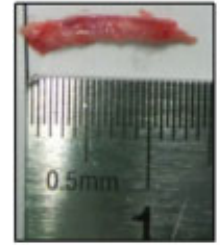

Sham

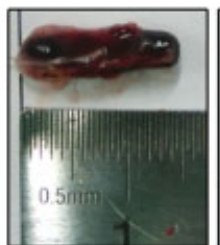

Model

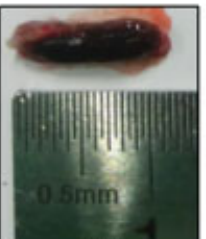

25

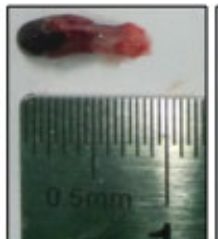

50

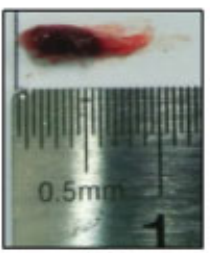

75
A

$\operatorname{RES}(\mathbf{m g} / \mathbf{k g})$

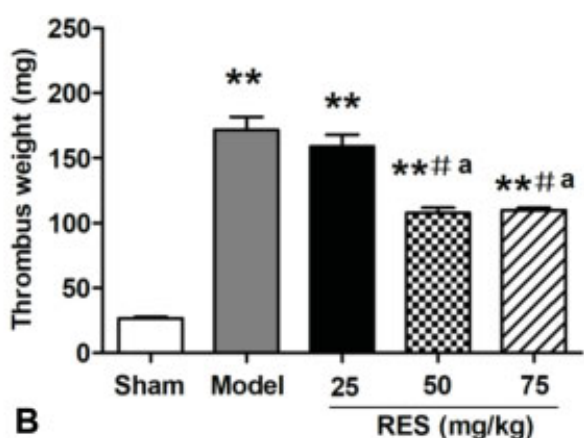

Fig. 5 Resveratrol (RES) alleviated thrombus formation in rats of inferior vena cava (IVC) stenosis-induced deep vein thrombosis (DVT). RES was intra-gastrically administered at 25,50 and $75 \mathrm{mg} / \mathrm{kg}$ once daily for 2 days. The first dose was administered at 1 hour before thrombus induction, and the second one was at 24 hours after surgery. One hour after the final administration, IVCs were harvested and weighed. (A) One representative IVC of each group and (B) thrombus weight are shown. Data were expressed as mean \pm standard error of the mean (SEM), $n=8$. ${ }^{* *} p<0.01$ versus sham group; ${ }^{*} p<0.05$ versus model group; ${ }^{a} p<0.05$ versus $25 \mathrm{mg} / \mathrm{kg}$ RES group.

suppressed thrombus-associated inflammation rather than surgery-associated inflammation. EX527, a potent and selective SIRT1 inhibitor, abolished the protective effect of RES on DVT (-Fig. 8), which further indicates that down-regulation of SIRT1 plays a critical role in DVT.
Our results showed that RES (agonist of SIRT1) increased SIRT1 protein expression, and EX527 (antagonist of SIRT1) reversed RES-induced increase of SIRT1 protein expression. Consistent with our findings, RES increases SIRT1 protein in other studies, attenuating anoxia/re-oxygenation-induced 


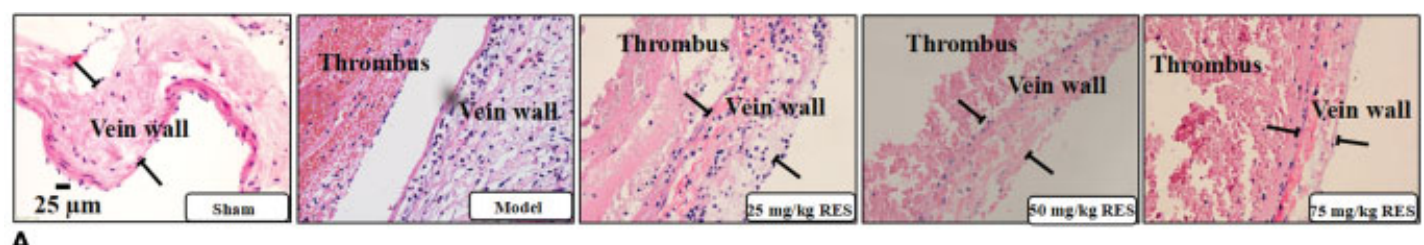

A
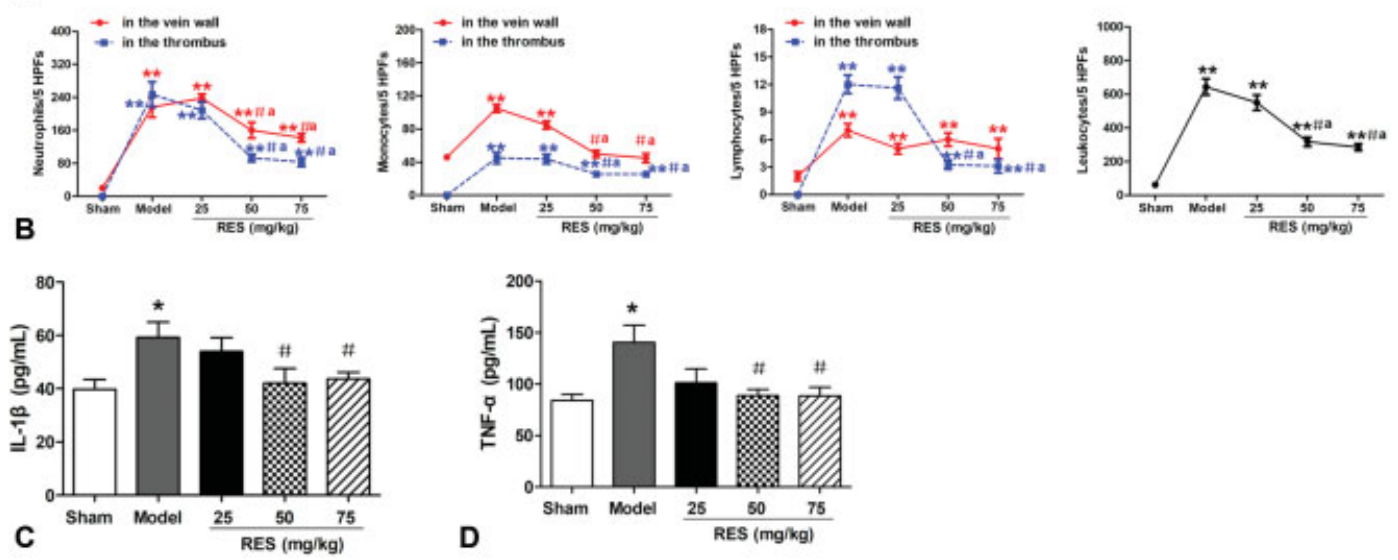

Fig. 6 Resveratrol (RES) alleviated infiltration of leukocytes and serum levels of interleukin-1 $\beta$ (IL-1 $\beta$ ) and tumour necrosis factor- $\alpha$ (TNF- $\alpha$ ). RES was intragastrically administered at 25,50 and $75 \mathrm{mg} / \mathrm{kg}$ once daily for 2 days. The first dose was administered at 1 hour before thrombus induction, and the second one was at 24 hours after surgery. One hour after the final administration, inferior vena cavas (IVCS) were collected. (A) Representative haematoxylin and eosin (H\&E) staining of IVC in each group. Scale bar $=25 \mu \mathrm{m}$. (B) Numbers of neutrophils, monocytes and lymphocytes in the vein wall and thrombus, and total numbers of leukocytes in both the vein wall and thrombus are shown. (C,D) Whole blood samples were collected and centrifuged to obtain serum for determining levels of IL-1 $\beta$ and TNF- $\alpha$. Data were expressed as mean \pm standard error of the mean (SEM), $n=8 .{ }^{*} p<0.05$ and ${ }^{* *} p<0.01$ versus sham group; ${ }^{\#} p<0.05$ versus model group; ${ }^{a} p<0.05$ versus $25 \mathrm{mg} / \mathrm{kg}$ RES group.

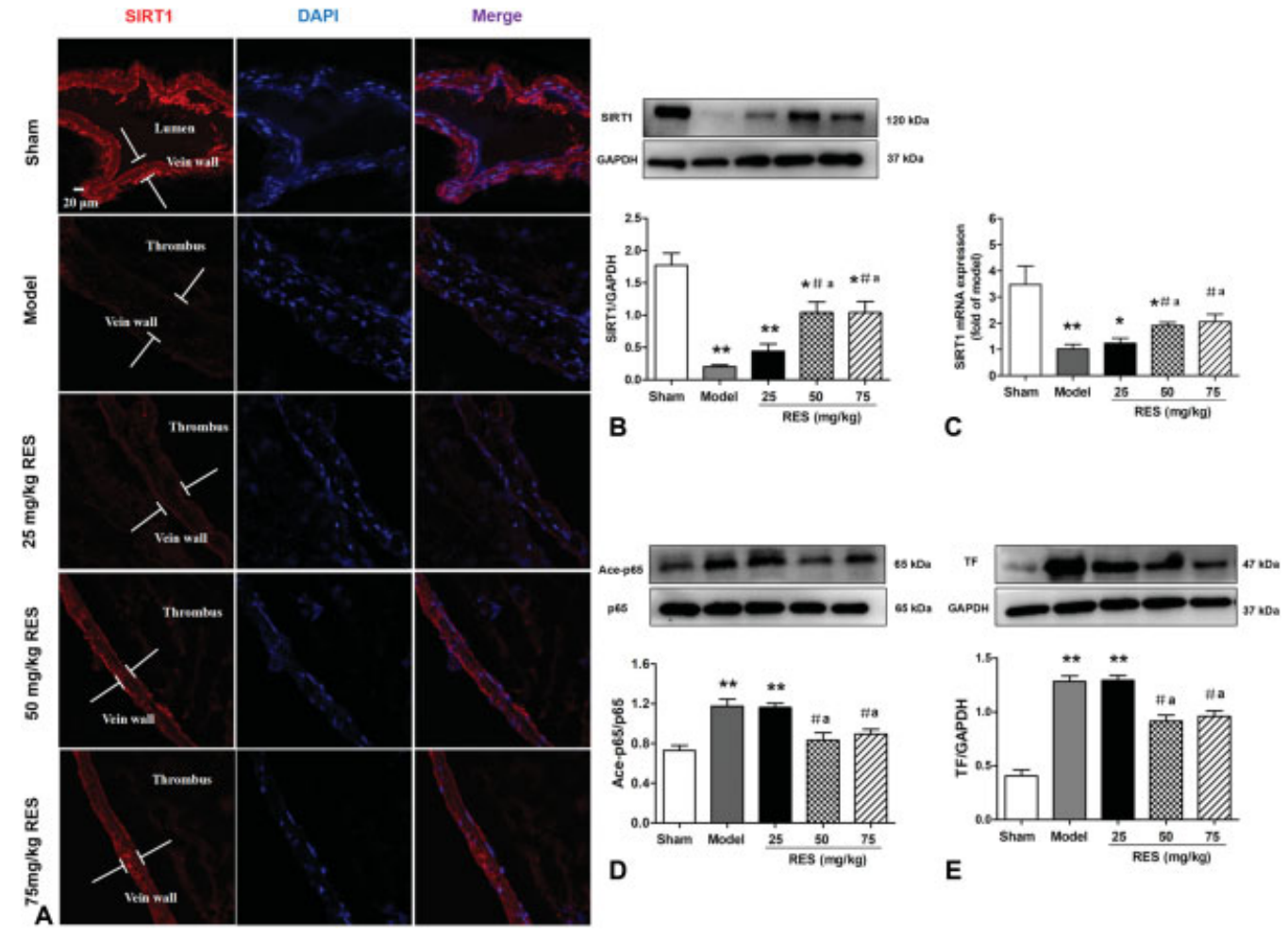

Fig. 7 Resveratrol (RES) increased protein and messenger ribonucleic acid (mRNA) expressions of Sirtuin 1 (SIRT1) and decreased protein expressions of acetylated p65 (Ace-p65) and tissue factor (TF). RES was intra-gastrically administered at 25, 50 and 75 mg/kg once daily for 2 days. The first dose was administered at 1 hour before thrombus induction, and the second one was at 24 hours after surgery. One hour after the final administration, tissue samples were collected. (A) Immunofluorescence analysis of SIRT1 (red) and 4',6 diamidino-2-phenylindole dihydrochloride (DAPI) (blue) in the thrombosed inferior vena cava (IVC). Scale bar $=20 \mu \mathrm{m}$. (B) SIRT1 protein expression was examined by Western blot. (C) SIRT1 mRNA expression was examined by quantitative real-time polymerase chain reaction (qRT-PCR). (D, E) Thrombosed IVC lysates were examined for Ace-p65 and TF protein expressions by Western blot. Data were expressed as mean \pm standard error of the mean (SEM), $n=8 .{ }^{*} p<0.05$ and ${ }^{* *} p<0.01$ versus sham group; ${ }^{*} p<0.05$ versus model group; ${ }^{a} p<0.05$ versus $25 \mathrm{mg} / \mathrm{kg}$ RES group. 

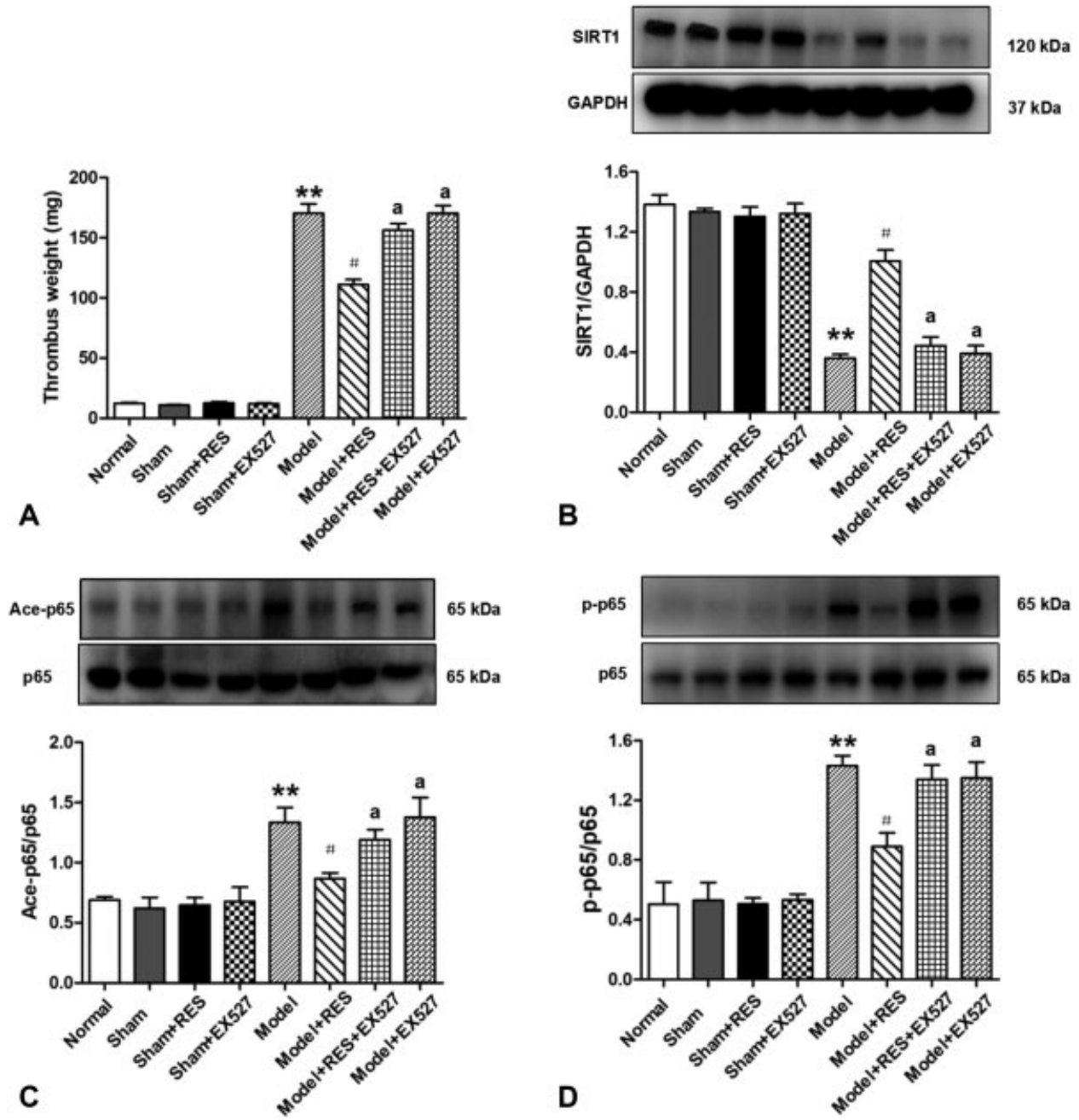

Fig. 8 EX527 abolished effects of resveratrol (RES) on thrombus weight and protein expressions of Sirtuin 1 (SIRT1), acetylated p65 (Ace-p65) and phosphorylated p65 (p-p65) at 1 day after inferior vena cava (IVC) stenosis in rats. RES was orally administered at $50 \mathrm{mg} / \mathrm{kg}$ once daily for 2 days. The first dose was administered at 1 hour before thrombus induction, and the second one was at 24 hours after surgery. EX527 was intraperitoneally injected at $10 \mathrm{mg} / \mathrm{kg}$ in a volume of $2 \mathrm{~mL} / \mathrm{kg}$ body weight 20 minutes before surgery. One hour after the final administration of RES, tissue samples were collected. (A) Thrombus weight is shown. (B-D) Thrombosed IVC lysates were examined for SIRT1, Ace-p65 and p-p65 protein expressions by Western blot. Data were expressed as mean \pm standard error of the mean (SEM), $n=6$. ${ }^{* *} p<0.01$ versus sham group; ${ }^{\#} p<0.05$ versus model group; ${ }^{a} p<0.05$ versus model + RES group.

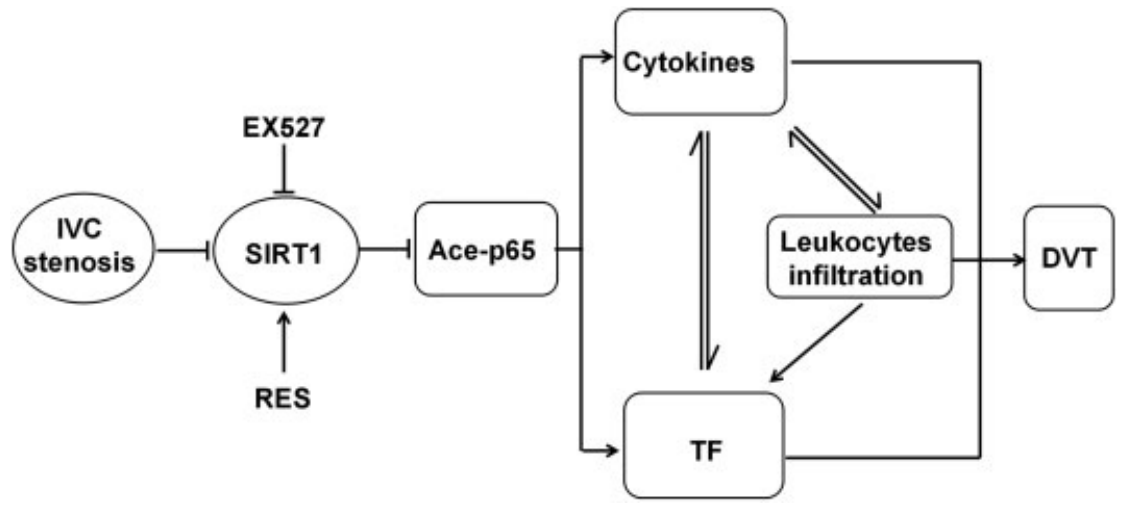

Fig. 9 Schematic diagram of how Sirtuin 1 (SIRT1) regulates inflammation in the process of inferior vena cava (IVC) stenosis-induced deep vein thrombosis (DVT). IVC stenosis reduces SIRT1 protein. Subsequently, acetylated p65 (Ace-p65) is increased, and then inflammatory cytokines and tissue factor (TF) are highly expressed from active leukocytes and endothelium. Besides, cytokines induce leukocytes and endothelial cells to express TF and vice versa. Cytokines promote leukocytes infiltration. Meanwhile, leukocytes infiltration promotes the secretion of cytokines and TF expression. All of these mediators contribute to DVT. 
mitochondria apoptosis in cardiomyocytes ${ }^{35}$ and acute kidney injury in a septic rat model, ${ }^{36}$ which is also reversed by EX527. The reason for RES and EX527 modulating protein expression of SIRT1 may be that RES (-Fig. 7C) and EX527 $7^{37,38}$ could regulate the transcription of SIRT1.

Therefore, our data showed that IVC stenosis down-regulated SIRT1 protein expression. Subsequently, Ace-p65 was increased, and then inflammatory cytokines and TF protein expression were highly expressed from active leukocytes and endothelium. Besides, cytokines induce leukocytes and endothelial cells to express TF protein and vice versa. ${ }^{27}$ Cytokines promote leukocytes infiltration. ${ }^{26}$ Meanwhile, leukocytes infiltration promotes the secretion of cytokines $^{23-25}$ and TF protein expression. All of these mediators contribute to DVT ( - Fig. 9).

\section{Conclusion}

Taken together, inflammation occurred and SIRT1 protein expression was remarkably decreased in rats of IVC stenosisinduced DVT. Besides, RES inhibited inflammation and DVT, while EX527 abolished these protective effects of RES. These results indicate that DVT is modulated by inflammation regulated via the SIRT1/NF-кB signalling pathway.

\section{What is known about this topic?}

- Inflammation plays a pivotal role in deep vein thrombosis (DVT).

- Sirtuin 1 (SIRT1) negatively regulated inflammation via deacetylating nuclear factor-kappa B (NK- кB) at Lys310.

\section{What does this paper add?}

- Inflammation biomarkers were significantly increased and SIRT1 was remarkable decreased in rats of DVT.

- RES inhibited inflammation and DVT with decreased deacetylation of NK- KB.

- EX527 abrogated the protective effect of RES on DVT.

\section{Authors' Contributions}

Baoqin Lin designed the research studies. Xaolan Yao performed the experiment and wrote the manuscript. Wenpei Chen, Jin Liu, Han Liu, Janis Y. Zhan, Shixia Guan, Ziqi Lu, Ping Tang and Peng Li analysed and interpreted data. All authors revised the manuscript and approved the final version.

\section{Funding}

This work was supported by Guangdong Public Welfare Research and Capacity Building Projects (2016A020217016).

\section{Conflict of Interest}

None declared.

\section{References}

1 Di Minno MN, Ambrosino P, Ambrosini F, Tremoli E, Di Minno G, Dentali F. Prevalence of deep vein thrombosis and pulmonary embolism in patients with superficial vein thrombosis: a systematic review and meta-analysis. J Thromb Haemost 2016;14 (05):964-972

2 Bawa H, Weick JW, Dirschl DR, Luu HH. Trends in deep vein thrombosis prophylaxis and deep vein thrombosis rates after total hip and knee arthroplasty. J Am Acad Orthop Surg 2018;26 (19):698-705

3 Glynn RJ, Ridker PM. Inflammation, venous thromboembolism, and what we can do about it. Eur Heart J 2018;39(39):3615-3617

4 Wang LM, Duan QL, Yang F, et al. Activation of circulated immune cells and inflammatory immune adherence are involved in the whole process of acute venous thrombosis. Int J Clin Exp Med 2014;7(03):566-572

5 Saghazadeh A, Rezaei N. Inflammation as a cause of venous thromboembolism. Crit Rev Oncol Hematol 2016;99:272-285

6 Engelmann B, Massberg S. Thrombosis as an intravascular effector of innate immunity. Nat Rev Immunol 2013;13(01):34-45

7 Schulz C, Engelmann B, Massberg S. Crossroads of coagulation and innate immunity: the case of deep vein thrombosis. J Thromb Haemost 2013;11(Suppl 1):233-241

8 Zhang Z, Hu L, Chen W, Zhou C, Gui G, Lin B. Danhong huayu koufuye prevents deep vein thrombosis through anti-inflammation in rats. J Surg Res 2016;201(02):340-347

9 Swystun LL, Liaw PC. The role of leukocytes in thrombosis. Blood 2016;128(06):753-762

10 Saha P, Humphries J, Modarai B, et al. Leukocytes and the natural history of deep vein thrombosis: current concepts and future directions. Arterioscler Thromb Vasc Biol 2011;31(03):506-512

11 Pikarsky E, Porat RM, Stein I, et al. NF-kappaB functions as a tumour promoter in inflammation-associated cancer. Nature 2004;431(7007):461-466

12 Malaponte G, Signorelli SS, Bevelacqua V, et al. Increased levels of NF-kB-dependent markers in cancer-associated deep venous thrombosis. PLoS One 2015;10(07):e0132496

13 Michan S, Sinclair D. Sirtuins in mammals: insights into their biological function. Biochem J 2007;404(01):1-13

14 Haigis MC, Guarente LP. Mammalian sirtuins-emerging roles in physiology, aging, and calorie restriction. Genes Dev 2006;20 (21):2913-2921

15 Liu TF, Vachharajani VT, Yoza BK, McCall CE. NAD+-dependent sirtuin 1 and 6 proteins coordinate a switch from glucose to fatty acid oxidation during the acute inflammatory response. J Biol Chem 2012;287(31):25758-25769

16 Yoshizaki T, Milne JC, Imamura T, et al. SIRT1 exerts anti-inflammatory effects and improves insulin sensitivity in adipocytes. Mol Cell Biol 2009;29(05):1363-1374

17 Breitenstein A, Stein S, Holy EW, et al. Sirt1 inhibition promotes in vivo arterial thrombosis and tissue factor expression in stimulated cells. Cardiovasc Res 2011;89(02):464-472

18 Hayashi H, Cherpokova D, Martinod K, et al. Sirt3 deficiency does not affect venous thrombosis or NETosis despite mild elevation of intracellular ROS in platelets and neutrophils in mice. PLoS One 2017;12(12):e0188341

19 de Lange P, Cioffi F, Senese R, et al. Nonthyrotoxic prevention of diet-induced insulin resistance by 3,5-diiodo-L-thyronine in rats. Diabetes 2011;60(11):2730-2739

20 Zhang H, Yan H, Zhou X, et al. The protective effects of Resveratrol against radiation-induced intestinal injury. BMC Complement Altern Med 2017;17(01):410

21 Nosaka M, Ishida Y, Kimura A, Kondo T. Time-dependent appearance of intrathrombus neutrophils and macrophages in a stasisinduced deep vein thrombosis model and its application to thrombus age determination. Int J Legal Med 2009;123(03): 235-240 
22 Esmon CT. Inflammation and thrombosis. J Thromb Haemost 2003;1(07):1343-1348

23 Levi M, van der Poll T. Two-way interactions between inflammation and coagulation. Trends Cardiovasc Med 2005;15(07): 254-259

24 Scaldaferri F, Sans M, Vetrano S, et al. Crucial role of the protein C pathway in governing microvascular inflammation in inflammatory bowel disease. J Clin Invest 2007;117(07):1951-1960

25 Szotowski B, Antoniak S, Poller W, Schultheiss HP, Rauch U. Procoagulant soluble tissue factor is released from endothelial cells in response to inflammatory cytokines. Circ Res 2005;96 (12):1233-1239

26 Nishimura S, Manabe I, Nagasaki M, et al. In vivo imaging visualizes discoid platelet aggregations without endothelium disruption and implicates contribution of inflammatory cytokine and integrin signaling. Blood 2012;119(08):e45-e56

27 Chu AJ. Tissue factor mediates inflammation. Arch Biochem Biophys 2005;440(02):123-132

28 Gillum MP, Kotas ME, Erion DM, et al. SirT1 regulates adipose tissue inflammation. Diabetes 2011;60(12):3235-3245

29 Cao L, Liu C, Wang F, Wang H. SIRT1 negatively regulates amyloidbeta-induced inflammation via the NF-кB pathway. Braz J Med Biol Res 2013;46(08):659-669

30 Li J, Li L, Wang S, et al. Resveratrol alleviates inflammatory responses and oxidative stress in rat kidney ischemia-reperfusion injury and H2O2-induced NRK-52E cells via the Nrf2/TLR4/NF-KB pathway. Cell Physiol Biochem 2018;45(04):1677-1689
31 Xu M, Cheng Z, Ding Z, Wang Y, Guo Q, Huang C. Resveratrol enhances IL-4 receptor-mediated anti-inflammatory effects in spinal cord and attenuates neuropathic pain following sciatic nerve injury. Mol Pain 2018;14:1744806918767549

32 Zhao H, Chen S, Gao K, et al. Resveratrol protects against spinal cord injury by activating autophagy and inhibiting apoptosis mediated by the SIRT1/AMPK signaling pathway. Neuroscience 2017;348:241-251

33 Timmers S, Auwerx J, Schrauwen P. The journey of resveratrol from yeast to human. Aging (Albany NY) 2012;4(03):146-158

34 Sin TK, Yung BY, Yip SP, et al. SIRT1-dependent myoprotective effects of resveratrol on muscle injury induced by compression. Front Physiol 2015;6:293

35 Tong Z, Xie Y, He M, et al. VDAC1 deacetylation is involved in the protective effects of resveratrol against mitochondria-mediated apoptosis in cardiomyocytes subjected to anoxia/reoxygenation injury. Biomed Pharmacother 2017;95:77-83

$36 \mathrm{Xu} \mathrm{S}$, Gao Y, Zhang Q, et al. SIRT1/3 activated by resveratrol attenuates acute kidney injury in a septic rat model. Oxid Med Cell Longev 2016;2016:7296092

37 Liu J, Han W, Chen L, Tang K. Mechanism of osteogenic and adipogenic differentiation of tendon stem cells induced by sirtuin 1. Mol Med Rep 2016;14(02):1643-1648

38 Guo H, Chen Y, Liao L, Wu W. Resveratrol protects HUVECs from oxidized-LDL induced oxidative damage by autophagy upregulation via the AMPK/SIRT1 pathway. Cardiovasc Drugs Ther 2013;27 (03):189-198 